OPEN ACCESS

Edited by:

Laszlo Csiba,

University of Debrecen, Hungary

Reviewed by:

Hrvoje Budincevic,

University Hospital Sveti Duh, Croatia

Nagy Zoltán,

Semmelweis University, Hungary

Ekaterina Titianova,

Military Medical Academy, Bulgaria

*Correspondence:

Tihamer Molnar

tihamermolnar@yahoo.com

Specialty section:

This article was submitted to

Stroke,

a section of the journa

Frontiers in Neurology

Received: 11 February 2021 Accepted: 20 May 2021

Published: 18 June 2021

Citation:

Schrick D, Ezer E, Tokes-Fuzesi M, Szapary L and Molnar T (2021) Novel

Predictors of Future Vascular Events in

Post-stroke Patients - A Pilot Study.

Front. Neurol. 12:666994.

doi: 10.3389/fneur.2021.666994

\section{Novel Predictors of Future Vascular Events in Post-stroke Patients - A Pilot Study}

\author{
Diana Schrick ${ }^{1}$, Erzsebet Ezer ${ }^{1}$, Margit Tokes-Fuzesi ${ }^{2}$, Laszlo Szapary ${ }^{3}$ and \\ Tihamer Molnar ${ }^{1 *}$ \\ ${ }^{1}$ Department of Anesthesiology and Intensive Care, Medical School, University of Pécs, Pécs, Hungary, ${ }^{2}$ Department of \\ Laboratory Medicine, Medical School, University of Pécs, Pécs, Hungary, ${ }^{3}$ Department of Neurology, Medical School, \\ University of Pécs, Pécs, Hungary
}

Introduction: A modified platelet function test (MPFT) was recently found to be superior compared to impedance aggregometry for selection of post-stroke patients with high on-treatment platelet reactivity (HTPR). We aimed to explore some peripheral blood cell characteristics as predictors of recurrent ischemic episodes. The predictive value of mPFT was also assessed in a cohort followed up to 36 months regarding recurrent ischemic vascular events.

Methods: As a novelty, not only whole blood (WB), but after 1-h gravity sedimentation the separated upper (UB) and lower half blood (LB) samples were analyzed including neutrophil antisedimentation rate (NAR) in 52 post-stroke patients taking clopidogrel. Area under the curve (AUC, $A \cup C_{\text {upper }}$ and $A \cup C_{\text {lower }}$, respectively) was separately measured by Multiplate in the WB, UB and LB samples to characterize ex vivo platelet aggregation in the presence of ADP. Next, the occurrence of vascular events (stroke, acute coronary syndrome, ACS) were evaluated during 36-month follow-up.

Results: A total of 11 vascular events (stroke $n=5$, ACS $n=6$ ) occurred during the follow-up period. The $\mathrm{AUC}_{\text {upper }}$ was significantly higher in patients with recurrent stroke

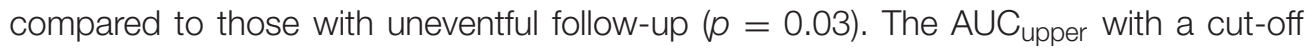
value $\geq 70$ based on the $\mathrm{mPFT}$, was able to predict all stroke events $(p=0.01)$, while the total vascular events were independently predicted by NAR with a sensitivity of $82 \%$ and specificity of $88 \%$.

Conclusions: A combination of NAR reflecting the inflammatory state and $\mathrm{AUC}_{\text {upper }}$ indicating HTPR may provide a better prediction of recurrent ischemic events suggesting a better selection of patients at risk, thus providing an individually tailored vascular therapy.

Keywords: recurrent stroke, vascular event, platelet function, platelet reactivity, outcome

\section{INTRODUCTION}

Despite successful recanalization strategies either with thrombolysis or using endovascular treatments for acute ischemic stroke, the eventual outcome of patients is far from desirable (1). Among many factors, some peripheral blood cells may play a pivotal role in post-procedural microcirculatory alterations contributing to the outcome $(2,3)$. A higher incidence of recurrent 
cerebral ischemia was described in post-stroke patients with high on-treatment platelet reactivity $(\operatorname{HTPR})(4,5)$. Numerous tests assessing ex vivo platelet reactivity were used for identification of patients at risk for HTPR (6). However, the prevalence of HTPR was shown to vary depending on the definition and assay used (7). A modified platelet function test (mPFT) was recently found to be superior compared to conventional Multiplate Electrode Aggregometry for selection of post-stroke patients with HTPR (8).

Therefore, we aimed to explore some peripheral blood cell characteristics including platelets and neutrophils as predictors of recurrent ischemic episodes and factors contributing to the outcome. The predictive value of the mPFT as a point-of-care test (POCT) was also compared to conventional Multiplate Electrode Aggregometry in a cohort followed up to 36 months regarding recurrent ischemic vascular events.

\section{MATERIALS AND METHODS}

\section{Subjects}

The study protocoll was approved by the University of Pecs Clinical Centre Regional and Institutional Research Ethics Comittee (8). Written informed consent was obtained from each patient. A total of 52 patients (age: $66 \pm 8$ years, male: 31) on antiplatelet therapy ( $75 \mathrm{mg}$ clopidogrel once daily) due to secondary stroke prevention were prospectively recruited into this study. The selected patients with previous anterior circulation large artery atherothrombosis were on regular medical check-up at the Outpatient Clinic of the Department of Neurology. Fasting venous blood samples were taken via a $21 \mathrm{G}$ peripheral venous canula from each patient and healthy subjects. Patients were instructed to take their daily clopidogrel at least $2 \mathrm{~h}$ prior to blood sampling. Exclusion criteria were acute infection and acute vascular events, such as acute ischemic stroke (AIS), transient ischemic attack (TIA), acute myocardial infarction (AMI), acute coronary syndrome (ACS), thrombocytopenia (platelet count $<150 \mathrm{G} / \mathrm{l}$ ), congenital platelet abnormalities, congenital disorder of haemostatis (e.g., hemophilia), anemia and patients on medical therapy influencing blood coagulation (e.g., oral anticoagulants, novel oral anticoagulants, non-steroid antiinflammatory drugs). The comorbidities, medications and smoking status were also recorded. Besides, the baseline erythrocyte sedimentation rate (ESR), C-reactive protein (CRP), and total blood count were measured. Next, the incidence of vascular events (ACS and recurrent ischemic stroke) in the total study population was evaluated in a 36 -month follow-up. ACS was defined by using the ACC/AHA guidelines (shortly: based on clinical history, ECG results, levels of cardiac markers, and the results of stress testing). Each recurrent ischemic stroke was confirmed by neuroimaging (CT or MRI). All patients with either ACS or recurrent ischemic stroke were presented at the Emergency Department and underwent a careful clinical evaluation then archived by an electronic database.

\section{Blood Sampling}

Venopuncture was performed from the cubital vein after short time strangulation of the arm with $21 \mathrm{G} B D$ vacutainer needle.
The total blood count was measured after taking into vacutainers with EDTA (REF: 368856, 5.4md EDTA). Whole blood for platelet aggregometry was also taken into hirudin containing tube for Multiplate Electrode Aggregometry.

\section{Platelet Antisedimentation Rate, Neutrophil Antisedimentation Rate}

Modified whole blood gravity sedimentation technique was developed for studying platelet and neutrophil sedimentation properties (8). After 1-h gravity sedimentation, the upper and lower half of the venous blood column was separately removed from the EDTA sedimentation tube and transferred to another EDTA tube for further analysis. An automatic cell counter system (Sysmex XN 9000, Sysmex Co, Japan, 2017) was applied to measure the upward floating (ascending) and sinking (nonascending) cells in the separated samples. Next, the platelet antisedimentation rate (PAR, \%), leukocyte antisedimentation rate (LAR,\%) and neutrophil antisedimentation rate (NAR, \%) were, respectively, calculated based on the equation:

$$
\frac{\text { cell count }_{\text {upper }}-\text { cell count }_{\text {lower }}}{\text { cell count }_{\text {upper }}+\text { cell count }_{\text {lower }}} \text { X100 }
$$

\section{Multiplate Electrode Aggregometry}

Platelet function test in the whole blood was perfomed from a hirudin containing tube with a Multiplate ${ }^{\circledR}$ Analyzer (Roche Diagnostics, Mannheim, Germany). Another hirudin containing tube was used for sedimentation, similarly to whole blood sedimentation in the EDTA-tube. After 1-h gravity sedimentation, the blood coloumn was devided into upper and lower samples. Platelet aggregometry was uniformly performed 60 min after blood sampling using adenosine diphosphate (ADP; $6.5 \mathrm{M}$ ) as agonist. As a novelty, not only whole blood, but after 1 -h gravity sedimentation the separated upper and lower half blood samples were simultaneously analyzed in each post-stroke patient taking clopidogrel. Aggregation level was expressed as the area under the curve (AUC). AUC was calculated by a Multiplate $^{\circledR}$ Analyzer using the product of aggregation unit $(\mathrm{AU}) \times$ time (minutes) (9). After ADP stimulation, the normal aggregation range was expected as AUC: 53-122 according to the manufacturer (9). Based on the whole blood AUC, patients on clopidogrel were categorized as responder cases with AUC $<53$ and resistant cases representing HTPR with an AUC $\geq 53$ (10).

\section{Statistical Analysis}

Data were evaluated using SPSS software package (Version 19.0, SPSS Inc, Chicago, USA). Categorical data were summarized by means of absolute and relative frequencies (counts and percentages). Quantitative data were presented as median and 25 th -75 th percentiles, as well as mean \pm SD. The KolmogorovSmirnov test was applied to check for normality. Chi-square test for categorical data and Student- $t$ test for continuous data were used for analysis of demographic and clinical factors. Nonparametric Mann-Whitney $U$ test was used for not normally distributed parameters. Correlation analysis was performed calculating Spearman's correlation coefficient (rho). A $p$-value $<0.05$ was considered statistically significant. 
TABLE 1 | Demography and clinical data of the total population, and comparison between patients without vs. with recurrent vascular events during 36-month follow-up.

\begin{tabular}{|c|c|c|c|c|}
\hline & $\begin{array}{c}\text { Total } \\
\text { population } \\
n=52\end{array}$ & $\begin{array}{c}\text { Uneventful } \\
n=41\end{array}$ & $\begin{array}{l}\text { Vascular } \\
\text { events } \\
n=11\end{array}$ & $p$-value \\
\hline age & $66 \pm 8$ & $66 \pm 8$ & $66 \pm 9$ & 0.937 \\
\hline male, $n$ & 34 & 26 & 8 & 0.564 \\
\hline hypertension, $n$ & 51 & 40 & 11 & 0.601 \\
\hline diabetes mellitus, $n$ & 14 & 10 & 4 & 0.427 \\
\hline smoking, $n$ & 11 & 9 & 2 & 1.000 \\
\hline ESR & $12(8-18)$ & $10(8-16)$ & $18(14-29)$ & 0.063 \\
\hline CRP & $1.9(0.7-4.6)$ & $1.8(0.7-5.0)$ & $2.2(1.4-3.35)$ & 0.614 \\
\hline PLT & 224 (200-260) & $224(207-251)$ & $243(171-300)$ & 0.805 \\
\hline PAR & $\begin{array}{c}67.9 \\
(63.1-73.4)\end{array}$ & $\begin{array}{c}67.8 \\
(62.9-73.5)\end{array}$ & $\begin{array}{c}70.0 \\
(64.6-72.6)\end{array}$ & 0.614 \\
\hline WBC & $6.8(5.8-8.0)$ & $6.6(5.8-7.9)$ & $7.4(5.5-10.6)$ & 0.420 \\
\hline LAR & $\begin{array}{c}35.7 \\
(23.7-46.3)\end{array}$ & $\begin{array}{c}36.2 \\
(24.7-46.4)\end{array}$ & $\begin{array}{c}34.4 \\
(24.0-43.5)\end{array}$ & 0.806 \\
\hline neutrophil & $\begin{array}{c}61.8 \\
(55.4-66.4)\end{array}$ & $62(56-67)$ & $58(51-62)$ & 0.317 \\
\hline NAR & $\begin{array}{c}-1.1 \\
(-4.8-6.5)\end{array}$ & $0.9(-3.9-7.2)$ & $\begin{array}{c}-5.2 \\
{[-6.8-(-4.7)]}\end{array}$ & 0.001 \\
\hline
\end{tabular}

Vascular events, recurrent stroke, and de novo acute coronary event; ESR, erythrocyte sedimentation rate; CRP, C-reactive protein; PLT, platelet; PAR, platelet antisedimentation rate; $W B C$, white blood cell; $L A R$, leukocyte antisedimentation rate; NAR, neutrophil antisedimentation rate. Data are presented as median and 25th-75th percentiles, except age as mean $\pm S D$.

\section{RESULTS}

A total of 52 convalescent ischemic stroke patients were prospectively enrolled into this pilot study. All patients have been previously suffered from large vessel occlusion. The demography and clinical data of the study population is summarized in Table 1. A total of 11 vascular events (stroke $n=5$, ACS $n=6$ ) occured during 36-month follow-up. Of the antisedimentation rate indices, only NAR showed significant difference between "uneventful" vs. "vascular events" groups (Table 1). It is noteworthy that no difference was observed between the baseline blood count parameters (platelet, leukocyte, neutrophil), while a trend-like difference was observed in the ESR (Table 1). The AUC in the whole blood, and in the upper and lower samples after 1 -h gravity sedimentation in the total population, and also a comparison between uneventful vs. stroke + ACS as well as uneventful vs. recurrent stroke alone subgroups are shown in (Table 2). The $\mathrm{AUC}_{\text {upper }}$ was significantly higher in patients with recurrent stroke compared to those with uneventful follow-up ( $p$ $=0.03)$ (Table 2).

\section{Independent Predictors}

Based on ROC analysis, the $\mathrm{AUC}_{\text {upper }}$ with a cut-off value $\geq 70$ measured by the mPFT was able to predict recurrent stroke events $(p=0.01)$ with the best sensitivity and specificity. Moreover, the total vascular events (stroke+ACS) was independently predicted by NAR with a sensitivity of $82 \%$ and
TABLE 2 | Area under the curve (AUC) in the whole blood, and AUC in the upper and lower samples after 1-h gravity sedimentation in the total population and comparison between uneventful vs. stroke + ACS as well as uneventful vs. recurrent stroke subgroups.

\begin{tabular}{|c|c|c|c|c|}
\hline & $\begin{array}{c}\text { Total } \\
\text { population } \\
n=52\end{array}$ & $\begin{array}{c}\text { Uneventful } \\
n=41\end{array}$ & $\begin{array}{c}\text { Stroke + ACS } \\
n=11\end{array}$ & $p$-value \\
\hline AUC & $40.5(27-53.5)$ & $40(27-54)$ & $42(32.5-44)$ & 0.866 \\
\hline$A \cup C_{\text {upper }}$ & $56(22.5-76.5)$ & 51.5 (19.5-77.5) & 65 (42-75.5) & 0.247 \\
\hline \multirow[t]{2}{*}{$\mathrm{AUC}_{\text {lower }}$} & $18(13.5-22)$ & $18(14-23)$ & $17(13-20)$ & 0.567 \\
\hline & $\begin{array}{c}\text { Total } \\
\text { population } \\
n=52\end{array}$ & $\begin{array}{c}\text { Uneventful } \\
n=41\end{array}$ & $\begin{array}{c}\text { Stroke } \\
\text { events } \\
n=5\end{array}$ & $p$-value \\
\hline AUC & $40.5(27-53.5)$ & $39(27-53)$ & $43(42-44)$ & 0.347 \\
\hline AUC upper & $56(22.5-76.5)$ & $49(21-74)$ & $77(71-92)$ & 0.020 \\
\hline $\mathrm{AUC}_{\text {lower }}$ & 18 (13.5-22) & $18(14-22)$ & $17(11-19)$ & 0.763 \\
\hline
\end{tabular}

AUC, area under the curve measured by Multiplate analyzer; $A \cup C_{\text {upper }}$, AUC in the upper sample after 1-h gravity sedimentation; AUClower, AUC in the lower sample after 1-h gravity sedimentation.

TABLE 3 | Predictors of vascular events during 36-month follow-up.

\begin{tabular}{lccccc}
\hline & $\boldsymbol{\beta}$ & $\boldsymbol{p}$-value & OR & \multicolumn{2}{c}{$\mathbf{9 5 \%} \mathbf{C l}$} \\
\hline age & -0.071 & 0.353 & 0.931 & 0.801 & 1.082 \\
AUC & -0.046 & 0.320 & 0.955 & 0.871 & 1.046 \\
AUC & -0.083 & 0.031 & 1.086 & 1.007 & 1.171 \\
NAR & -0.489 & 0.032 & 0.613 & 0.392 & 0.960
\end{tabular}

$A \cup C$, area under the curve measured by Multiplate analyzer; $A \cup C_{\text {upper, }} A \cup C$ in the upper sample after 1-h gravity sedimentation; NAR, neutrophil antisedimentation rate; OR, odds ratio, 95\% Cl, 95\% confidence interval. Binary logistic regression analysis.

specificity of $88 \%$ using a multiple regression analysis including relevant covariates (Table 3). Neither recurrent stroke nor ACS showed association with HTPR status defined by AUC $>53$ measured by the Multiplate in the whole blood.

\section{Cut-Off Values of Predictors}

The ROC curves of variables predicting recurrence of vascular events during follow-up are shown in Figure 1. In this cohort, NAR with a cut-off $\geq-0.431$ independently predicted recurrence of total vascular events (stroke $+\mathrm{ACS}, n=11$ ) with a sensitivity of $82 \%$ and specificity of $88 \%$ during 36 -month follow-up (Area: $0.847, p=0.002$, 95\%CI: 0.703-0.992) (Figure 1A). Furthermore, ROC of platelet function test based on impedance aggregometry in the upper blood sample after 1-h gravity sedimentation revealed, that $\mathrm{AUC}_{\text {upper }}$ with a cut-off $\geq 70$ predicted recurrent stroke with a sensitivity of $80 \%$ and specificity of $74 \%$ during 36-month follow-up (Area:0.813, $p=0.023$, 95\%CI:0.689-0.937) (Figure 1B). Finally, a more precise model was created, when a ROC analysis was performed with predicted probablity of the combination of NAR and PFT upper (Area:0.881, $p=0.001$, 95\%CI:0.754-1.0) (Figure 1C). 

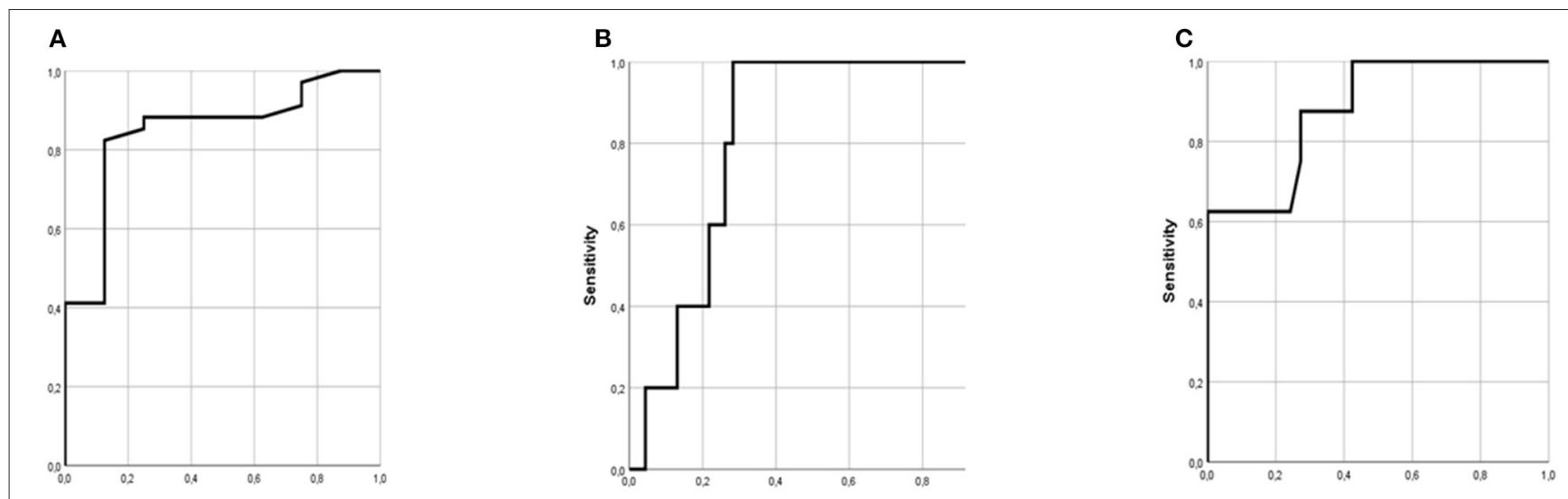

FIGURE 1 | ROC curves of variables predicting recurrence of vascular events during follow-up. (A) ROC of neutrophil antisedimentation rate (NAR) (Area: $0.847, p=$ 0.002, 95\% Cl: 0.703-0.992). (B) ROC of platelet function test based on impedance aggregometry in the upper blood sample (AUC ${ }_{\text {upper }}$ ) after 1-h gravity

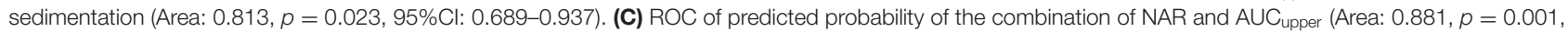
95\%Cl: 0.754-1.0).

\section{DISCUSSION}

Activation of neutrophils reflected by NAR was shown here as the most sensitive marker of recurrence of ischemic cerebral episodes in post-stroke patients taking clopidogrel. Both, animal and clinical data support the pivotal role of activated peripheral blood cells (e.g., neutrophils, monocytes, platelets) in neuroinflammation due to ischemic stroke (2, 3, 11). One side, the dynamic microcirculatory stall phenomenon in the hyperacute stage can be a contributing factor to ongoing penumbral brain injury $(2,12)$, on the other side the sustained detrimental effects of activated leukocytes in the systemic circulation carries a constant risk in patients with chronic inflammatory state (e.g., vascular diseases) (13). Interestingly, a downward motion of neutrophils during 1-h gravity sedimentation expressed by a negative value of NAR was observed in those patients who suffered from composite vascular events during 36-month follow-up. In contrast, an upward motion of both, leukocytes and platelets proportionally to their activation was described previously in acute ischemic stroke (3), post-stroke infection (14) and burn patients (15). Neither LAR, nor PAR was found to be predictive for future vascular events in convalescent stroke patients suggesting that leukocytes and platelets exert their actions predominantly in the acute phase of stroke. Our finding also suggests that neutrophils are important markers of stroke outcome as their predictive role was recently shown in patients with acute coronary syndrome (16).

Numerous data highlight that a high proportion of patients with cardiovascular diseases have ex vivo HTPR on their prescribed antiplatelet regimen $(4,5,7)$. Although several studies show an increased rate of recurrent cerebrovascular ischemic events in patients presenting HTPR, the diagnostics of HTPR has been unsolved so far $(4,17)$. Here, the state of clopidogrel resistance based on Multiplate electrode aggregometry from the whole blood was not able to predict recurrent stroke. However, a higher AUC ( $\geq 70$ as a cut-off value) from the separated upper blood sample after 1-h gravity sedimentation emerged as a novel independent predictor of future stroke episode in our study. This observation suggests that the upward motion of platelets might be associated with increased thrombotic tendency. Further studies are needed to explore the characteristics of this subpopulation of platelets and their impact on post-stroke complications and outcome. When the combination of NAR and PFT $_{\text {upper }}$ was used in the statistical model, the predicted probability of a future vascular event was even more accurate.

In summary, while $\mathrm{AUC}_{\text {upper }}$ indicates more precise definition of HTPR, NAR rather reflects the inflammatory state in poststroke patients (18). Based on this small, single-center pilot study, these novel markers may provide a better prediction of recurrent ischemic events leading to a better selection of patients at risk and providing an individually tailored vascular therapy including antiplatelet and anti-inflammatory regimens $(17,19)$.

\section{LIMITATIONS}

This is a small prospective cohort with a 36-month followup focusing primarily on recurrent coronary and cerebral ischemic episodes which required hospitalization. However, the silent ischemic lesion recurrence on MRI was not explored here. Therefore, a large, adequately sized, prospective multicenter study is needed to determine whether these novel assessments of HTPR in conjunction with pharmacogenetic and neuroimaging (diffusion weighted imaging, DWI) data, improves our ability to predict the risk of recurrent vascular events in patients with cardiovascular diseases. Although the interaction between inflammation and ischemic stroke is multifaceted, a better understanding of such mechanisms may lead to enhanced secondary prevention including immunomodulatory approaches and more precise antiplatelet therapy $(20,21)$. 


\section{DATA AVAILABILITY STATEMENT}

The raw data supporting the conclusions of this article will be made available by the authors, without undue reservation.

\section{ETHICS STATEMENT}

The studies involving human participants were reviewed and approved by Local Ethics Committee of the University of Pécs. The patients/participants provided their written informed consent to participate in this study.

\section{REFERENCES}

1. Bhaskar S, Stanwell P, Cordato D, Attia J, Levi C. Reperfusion therapy in acute ischemic stroke: dawn of a new era? BMC Neurol. (2018) 18:8. doi: 10.1186/s12883-017-1007-y

2. Erdener SE, Tang J, Kiliç K, Postnov D, Giblin JT, Kura S, et al. Dynamic capillary stalls in reperfused ischemic penumbra contribute to injury: a hyperacute role for neutrophils in persistent traffic jams. J Cereb Blood Flow Metab. (2020) 41:236-52. doi: 10.1177/0271678X20914179

3. Molnar T, Peterfalvi A, Szereday L, Pusch G, Szapary L, Komoly S, et al. Deficient leukocyte antisedimentation is related to post-stroke infections and outcome. J Clin Pathol. (2008) 61:1209-13. doi: 10.1136/jcp.2008.059840

4. Fiolaki A, Katsanos AH, Kyritsis AP, Papadaki S, Kosmidou M, Moschonas IC, et al. High on treatment platelet reactivity to aspirin and clopidogrel in ischemic stroke: a systematic review and meta-analysis. J Neurol Sci. (2017) 376:112-6. doi: 10.1016/j.jns.2017.03.010

5. Rao Z, Zheng H, Wang F, Wang A, Liu L, Dong K, et al. The association between high on-treatment platelet reactivity and early recurrence of ischemic events after minor stroke or TIA. Neurol Res. (2017) 39:719-26. doi: 10.1080/01616412.2017.1312793

6. Kinsella JA, Tobin WO, Cox D, Coughlan T, Collins R, O’Neill D, et al. Prevalence of ex vivo high on-treatment platelet reactivity on antiplatelet therapy after transient ischemic attack or ischemic stroke on the PFA100(®) and VerifyNow(®). J Stroke Cerebrovasc Dis. (2013) 22:e84-92. doi: 10.1016/j.jstrokecerebrovasdis.2012.07.012

7. Lim ST, Coughlan CA, Murphy SJ, Fernandez-Cadenas I, Montaner J, Thijs $\mathrm{V}$, et al. Platelet function testing in transient ischaemic attack and ischaemic stroke: a comprehensive systematic review of the literature. Platelets. (2015) 26:402-12. doi: 10.3109/09537104.2015.1049139

8. Ezer E, Schrick D, Tokés-Füzesi M, Szapary L, Bogar L, Molnar T. A novel approach of platelet function test for prediction of attenuated response to clopidogrel. Clin Hemorheol Microcirc. (2019) 73:359-69. doi: $10.3233 / \mathrm{CH}-190580$

9. Kim J, Cho CH, Jung BK, Nam J, Seo HS, Shin S, et al. Comparative evaluation of plateletworks, multiplate analyzer and platelet function analyzer-200 in cardiology patients. Clin Hemorheol Microcirc. (2018) 70:25765. doi: 10.3233/CH-170331

10. Bonello L, Tantry US, Marcucci R, Blindt R, Angiolillo DJ, Becker R, et al. Consensus and future directions on the definition of high on-treatment platelet reactivity to adenosine diphosphate. J Am Coll Cardiol. (2010) 56:91933. doi: 10.1016/j.jacc.2010.04.047

11. Csecsei P, Pusch G, Ezer E, Berki T, Szapary L, Illes Z, et al. Relationship between cardiac troponin and thrombo-inflammatory molecules in prediction of outcome after acute ischemic stroke. J Stroke Cerebrovasc Dis. (2018) 27:951-6. doi: 10.1016/j.jstrokecerebrovasdis.2017.10.040

12. El Amki M, Glück C, Binder N, Middleham W, Wyss MT, Weiss T, et al. Neutrophils obstructing brain capillaries are a major cause of no-reflow in ischemic stroke. Cell Rep. (2020) 33:108260. doi: 10.1016/j.celrep.2020.108260

13. Elkind MS, Cheng J, Rundek T, Boden-Albala B, Sacco RL. Leukocyte count predicts outcome after ischemic stroke:

\section{AUTHOR CONTRIBUTIONS}

DS, EE, LS, and TM conceived, designed and coordinated the study, participated in acquisition, and interpretation of data. DS and TM drafted the manuscript. MT-F performed the laboratory measurements. MT-F and TM participated in the statistical analysis. All authors read and approved the manuscript.

\section{FUNDING}

The study was supported by EFOP-3.6.3-VEKOP-16-2017-00009 at the University of Pécs.

the Northern Manhattan stroke study. J Stroke Cerebrovasc Dis. (2004) 13:220-7. doi: 10.1016/j.jstrokecerebrovasdis.2004. 07.004

14. Molnar T, Papp V, Banati M, Szereday L, Pusch G, Szapary L, et al. Relationship between C-reactive protein and early activation of leukocytes indicated by leukocyte antisedimentation rate (LAR) in patients with acute cerebrovascular events. Clin Hemorheol Microcirc. (2010) 44:183-92. doi: 10.3233/CH-2010-1273

15. Loibl C, Rozanovic M, Bogár L, Pankaczi A, Kovács P, Miseta A, et al. Lack of early platelet and leukocyte activation can indicate complications after major burn injury. Clin Hemorheol Microcirc. (2020) 77:17-26. doi: 10.3233/CH-190779

16. Dong $\mathrm{CH}$, Wang ZM, Chen SY. Neutrophil to lymphocyte ratio predict mortality and major adverse cardiac events in acute coronary syndrome: a systematic review and meta-analysis. Clin Biochem. (2018) 52:131-6. doi: 10.1016/j.clinbiochem.2017.11.008

17. Yan AR, Naunton M, Peterson GM, Fernandez-Cadenas I, Mortazavi R. Effectiveness of platelet function analysis-guided aspirin and/or clopidogrel therapy in preventing secondary stroke: a systematic review and meta-analysis. J Clin Med. (2020) 9:3907. doi: 10.3390/jcm91 23907

18. Efe E, Kocayigit I, Türker PM, Murat K, Erkan A, Sedat T, et al. Plateletto-lymphocyte ratio but not neutrophil-to-lymphocyte ratio predicts high on-treatment platelet reactivity in clopidogrel-treated patients with acute coronary syndrome. Indian J Pharmacol. (2016) 48:355-9. doi: 10.4103/0253-7613.186205

19. Lee M, Saver JL, Hong KS, Rao NM, Wu YL, Ovbiagele B. Antiplatelet regimen for patients with breakthrough strokes while on aspirin: a systematic review and meta-analysis. Stroke. (2017) 48:2610-3. doi: 10.1161/STROKEAHA.117.017895

20. Shekhar S, Cunningham MW, Pabbidi MR, Wang S, Booz GW, Fan F. Targeting vascular inflammation in ischemic stroke: Recent developments on novel immunomodulatory approaches. Eur J Pharmacol. (2018) 833:531-44. doi: 10.1016/j.ejphar.2018.06.028

21. Ruhnau J, Schulze J, Dressel A, Vogelgesang A. Thrombosis, neuroinflammation, and poststroke infection: the multifaceted role of neutrophils in stroke. J Immunol Res. (2017) 2017:5140679. doi: $10.1155 / 2017 / 5140679$

Conflict of Interest: The authors declare that the research was conducted in the absence of any commercial or financial relationships that could be construed as a potential conflict of interest.

Copyright (c) 2021 Schrick, Ezer, Tokes-Fuzesi, Szapary and Molnar. This is an open-access article distributed under the terms of the Creative Commons Attribution License (CC BY). The use, distribution or reproduction in other forums is permitted, provided the original author(s) and the copyright owner(s) are credited and that the original publication in this journal is cited, in accordance with accepted academic practice. No use, distribution or reproduction is permitted which does not comply with these terms. 